\title{
Circulating bioactive bacterial DNA is associated with immune activation and complications in common variable immunodeficiency
}

\author{
Hsi-en Ho, ${ }^{1}$ Lin Radigan, ${ }^{1}$ Gerold Bongers, ${ }^{2}$ Ahmed El-Shamy, ${ }^{1}$ and Charlotte Cunningham-Rundles ${ }^{1}$ \\ 'Department of Medicine and ${ }^{2}$ Microbiome Translational Center, Precision Immunology Institute, Department of \\ Oncological Sciences, Icahn School of Medicine at Mount Sinai, New York, New York, USA.
}

Common variable immunodeficiency (CVID) is characterized by profound primary antibody defects and frequent infections, yet autoimmune/inflammatory complications of unclear origin occur in $50 \%$ of individuals and lead to increased mortality. Here, we show that circulating bacterial 165 rDNA belonging to gut commensals was significantly increased in CVID serum $(P<0.0001)$, especially in patients with inflammatory manifestations $(P=0.0007)$. Levels of serum bacterial DNA were associated with parameters of systemic immune activation, increased serum IFN- $\gamma$, and the lowest numbers of isotype-switched memory B cells. Bacterial DNA was bioactive in vitro and induced robust host IFN- $\gamma$ responses, especially among patients with CVID with inflammatory manifestations. Patients with X-linked agammaglobulinemia (Bruton tyrosine kinase [BTK] deficiency) also had increased circulating bacterial 165 rDNA but did not exhibit prominent immune activation, suggesting that BTK may be a host modifier, dampening immune responses to microbial translocation. These data reveal a mechanism for chronic immune activation in CVID and potential therapeutic strategies to modify the clinical outcomes of this disease.

Conflict of interest: The authors have declared that no conflict of interest exists.

Copyright: (c) 2021, Ho et al. This is an open access article published under the terms of the Creative Commons Attribution 4.0 International License.

Submitted: September 29, 2020

Accepted: September 1, 2021

Published: October 8, 2021

Reference information: /CI Insight. 2021;6(19):e144777.

https://doi.org/10.1172/jci.

insight.144777.

\section{Introduction}

Common variable immunodeficiency (CVID), the most prevalent symptomatic primary immunodeficiency, is characterized by low levels of serum IgG, IgA, and/or IgM, and a lack of production of specific antibodies (1-3). As with other primary immune defects, patients with CVID are susceptible to recurrent severe infections. Notably, however, at least $50 \%$ of patients with CVID develop additional inflammatory complications $(4,5)$. These noninfectious manifestations include autoimmunity, interstitial lung disease, enteropathy, nodular regenerative hyperplasia of the liver, systemic granulomatous disease, lymphoid hyperplasia, and lymphoid malignancy (4-7). These complications are a major clinical challenge because they are not substantially ameliorated by standard IgG replacement therapy. As a whole, inflammatory conditions lead to an estimated 11-fold increased morbidity and mortality in patients with CVID (5).

Recent investigations have identified genetic defects leading to loss of B cell development and other defects in immune regulation in $20 \%-25 \%$ of individuals, but the pathogenesis of inflammatory complications in CVID has remained unexplained in most cases $(8,9)$. We previously demonstrated marked upregulation of IFN-related pathways in these individuals with CVID by mRNA transcriptional profiling (10). This IFN signature also distinguished individuals with CVID who had inflammatory conditions from other individuals with CVID and from healthy controls. To identify the origin of this pathological IFN signature, we used mass cytometry and found an expanded population of innate lymphoid cells (ILCs) positive for IFN- $\gamma$, IL-17A, and IL-22 in the peripheral blood and gastrointestinal and lung tissues of individuals with CVID who had inflammatory complications (11). ILCs typically play an important role in host-commensal homeostasis (12), and their excessive activity and/or proliferation appear to contribute to systemic and organ-specific inflammation in CVID. However, the stimulus underlying these immune responses has remained unknown.

Humoral immunity contributes to anatomical containment of commensal organisms in experimental animal models (13). In mice, secretory IgA and IgM have been shown to limit bacterial translocation from mucosal compartments (14-17). Microbiota-specific IgG has been detected in mice and healthy 
humans, with evidence of direct binding to a subset of gut microbiota (18-20). When commensal organisms are not appropriately contained, bacterial translocation, defined as the translocation of bacteria and/or their products without overt bacteremia, has been shown to drive chronic inflammation in disease states, such as HIV and inflammatory bowel disease $(21,22)$. In this study, we evaluated the functional impact of primary antibody defects on host-commensal compartmentalization in patients with lack of antibody production. Specifically, we sought the presence of a conserved DNA sequence encoding bacterial 16S RNA (16S rDNA) in the serum of a large cohort of well-characterized patients with CVID and for comparison, patients with X-linked agammaglobulinemia (XLA). We also asked whether the circulating bacterial DNA could be a driver of inflammatory complications in CVID, serving as a critical immune stimulus for the pathological IFN- $\gamma$ signature found in inflammatory CVID.

\section{Results}

Raised circulating bacterial DNA in CVID and XLA. To assess the presence and magnitude of microbial translocation in CVID, we first employed quantitative PCR to measure bacterial 16S rDNA levels in serum samples obtained from 92 patients with CVID in their usual state of health and 26 healthy individuals. We also measured serum 16S rDNA levels from 15 patients with XLA to evaluate whether microbial translocation was a shared feature among patients with profound defects in humoral immunity (Table 1). Bacterial 16S rDNA levels were significantly elevated in CVID sera (mean 19.15 copies/L, range 0.4-237.4 copies/L) as compared with healthy controls (mean 5.99 copies/L, range $1.2-18.88$ copies/L, $P<0.0001$ ). Patients with XLA also had similarly elevated serum $16 \mathrm{~S}$ rDNA levels as compared with healthy controls (mean 16.34 copies/L, range 2.36-42.31 copies/L, $P=0.0008$ ) (Figure 1A). The size of bacterial 16S rDNA amplicons derived from the serum samples was approximately 254 nucleotides long, providing a minimal estimate of the circulating bacterial DNA size in the patients.

Increased serum-soluble CD14 and lipopolysaccharide-binding protein in CVID but not in XLA. We next assessed whether bacterial translocation, identified as elevated circulating bacterial DNA, was accompanied by systemic immune activation in CVID or XLA. We quantified serum-soluble CD14 (sCD14), which is secreted by monocytes/macrophages upon exposure to bacterial products, including CpG DNA, Gram-negative LPS, and Gram-positive bacterial components (23-25). We also measured serum lipopolysaccharide-binding protein (LBP), produced by hepatocytes in response to LPS stimulation (26). As compared with healthy controls, serum sCD14 levels were significantly increased in patients with CVID (mean $6670 \mathrm{ng} / \mathrm{mL}$ vs. $3846 \mathrm{ng} / \mathrm{mL}, P<0.0001$, Figure 1B). Similarly, serum LBP was significantly elevated in CVID compared with healthy controls (mean 17,906 ng/mL [CVID] vs. $8392 \mathrm{ng} / \mathrm{mL}$ [healthy controls], $P<0.0001$, Figure 1C). Among patients with CVID, serum sCD14 was positively associated with serum bacterial 16S rDNA (Spearman's $r=0.28, P=0.0166$, Supplemental Figure 1A; supplemental material available online with this article; https://doi.org/10.1172/jci.insight.144777DS1). There was also a positive association between SCD14 and LBP (Spearman's $r=0.21, P=0.0735$, Supplemental Figure 1B). However, in contrast to CVID, these systemic markers of immune activation were not significantly elevated in patients with XLA as compared with healthy controls (Figure 1, B and C).

Lack of detection of endotoxin in CVID serum. Endotoxin from Gram-negative bacteria has been reported in serum in a few studies of patients with CVID but not found in other studies (27-30). Confirming the latter reports, we did not detect endotoxin in the serum of our CVID cohort in either undiluted and diluted serum using 4 commercial assays (EndoLISA, Pierce LAL Chromogenic Endotoxin Quantitation kit, Limulus Amebocyte Lysate Chromogenic Endpoint assay, and ToxinSensor). Given that 90 out of 92 patients with CVID in this cohort were on IgG replacement therapy, we tested whether endotoxins could perhaps be bound by the infused IgG. By examining the binding specificity of i.v. Ig products, we found that the polyclonal IgG antibodies contained in these commercial products bound endotoxin in a dose-dependent manner, suggesting potential interference either within serum samples themselves and/or in the context of the detecting assays (Supplemental Figure 2).

Markers of mucosal epithelial barrier dysfunction in CVID. Next, we sought evidence of a dysfunctional mucosal barrier that, in addition to the loss of mucosal antibody, could contribute to the translocation of microbial products in CVID. Zonulin is a human protein positively associated with intestinal wall permeability through its role as modulator of intercellular tight junctions between epithelial cells (31-33). Zonulin levels in CVID serum were markedly elevated as compared with healthy controls (mean 18.71 $\mathrm{ng} / \mathrm{mL}$ vs. $6.99 \mathrm{ng} / \mathrm{mL}$, respectively, $P=0.0003$, Figure 2A). Additionally, we evaluated serum levels of 
Table 1. Characteristics of the study population

\begin{tabular}{|c|c|c|}
\hline & CVID & XLA \\
\hline Number of participants & 92 & 15 \\
\hline Female, $n(\%)$ & $52(54)$ & $0(0)$ \\
\hline Autoimmunity/inflammatory complications, $n$ (\%) & $70(76)$ & $0(0)$ \\
\hline \multicolumn{3}{|l|}{ Median lab values (range) } \\
\hline Diagnostic IgG (mg/dL) & 184 (UD-594) & UD (UD-133) \\
\hline White blood cells $\left(10^{3} / \mu \mathrm{L}\right)$ & $5.1(1.5-11.4)$ & $7.1(2.0-11.3)$ \\
\hline Total lymphocyte counts $(/ \mu \mathrm{L})$ & $1200(300-3700)$ & $1300(651-5974)$ \\
\hline CD4 $^{+} \mathrm{T}$ cells $(/ \mu \mathrm{L})$ & $534(182-2304)$ & $765(378-4315)$ \\
\hline CD8 $^{+} \mathrm{T}$ cells $(/ \mu \mathrm{L})$ & $291(14-1741)$ & $624(164-1456)$ \\
\hline CD19+ B cells $(/ \mu \mathrm{L})$ & $140(0-788)$ & $1(0-7)$ \\
\hline
\end{tabular}

UD, undetectable.

intestinal fatty-acid binding protein (I-FABP), an intestinal epithelium-specific protein that can leak into circulation in the setting of gut barrier dysfunction $(34,35)$. Here also, serum I-FABP levels were significantly elevated among patients with CVID compared with healthy controls (mean 3346 pg/mL vs. 1992 $\mathrm{pg} / \mathrm{mL}$, respectively, $P=0.0006$, Figure $2 \mathrm{~B}$ ). In contrast to the patients with CVID, neither serum zonulin nor I-FABP levels were significantly elevated among patients with XLA (Figure 2, A and B). When examining the clinical phenotypes of the CVID cohort, we found that serum zonulin and I-FABP levels were significantly elevated among patients with CVID regardless of the presence or absence of known enteropathy (Supplemental Figure 3, A and B).

The gastrointestinal tract is a major source of bacterial translocation in CVID. The gastrointestinal tract contains a large bacterial mass (36). We hypothesized that the gastrointestinal tract could be a principal source of bacterial translocation in antibody deficiency. To evaluate for this, we sequenced circulating 16S rRNA genes (rDNA) to identify the corresponding bacterial taxa. Serum $16 \mathrm{~S}$ rRNA gene profiling of serum of 25 patients with CVID revealed that the translocation of bacterial DNA was derived from a diverse range of commensal species, predominantly by gut-associated bacterial phyla, including Firmicutes, Bacteroidetes, Actinobacteria, and Proteobacteria (Figure 3A). Notably, DNA from gut commensal families previously reported to be highly coated by secreted antibodies (Lachnospiraceae, Ruminococcaceae, Erysipelotrichaceae, Bacteroidaceae, and Comamonadaceae) in healthy humans were found in high abundance in the sera of these patients with CVID (Figure 3B) (37-40). All bacterial families detected and ranked by taxonomic abundance are tabulated in Supplemental Table 1.

Translocation of bacterial DNA is associated with inflammatory manifestations in CVID. We then asked whether bacterial translocation, specifically higher levels of serum bacterial 16S rDNA levels, was associated with the inflammatory manifestations in CVID. Indeed, patients with CVID with inflammatory complications had significantly higher serum bacterial DNA levels (mean 22.15 copies/L, range 0.4-237.4 copies/L) as compared with CVID individuals without such conditions (mean 9.59 copies $/ \mathrm{L}$, range 1.14-38.46 copies/L, $P=0.0007$, Figure 4A). Serum sCD14 levels were also significantly elevated in patients with CVID who had inflammatory complications compared with those without such complications (mean $6829 \mathrm{ng} / \mathrm{mL}$ vs. $6144 \mathrm{ng} / \mathrm{mL}$, respectively, $P=0.027$, Figure $4 \mathrm{~B}$ ) but the differences were smaller. The mean serum LBP levels were also higher in those with inflammatory manifestations, though this did not reach statistical significance (Figure 4C).

Bacterial translocation and immunological parameters. We then evaluated whether specific host immune parameters were associated with elevated circulating bacterial DNA. First, bacterial 16S rDNA levels were not correlated with blood IgG levels in CVID or patients with XLA, either at diagnosis (baseline) or with Ig replacement (not shown). In this CVID cohort, where serum IgA and IgM were severely reduced in most patients, we found that serum $16 \mathrm{~S}$ rDNA was also not related to serum IgA or IgM levels (not shown). However, for patients with CVID, those with severely reduced isotype-switched 
A

\begin{tabular}{l}
$A$ \\
\hline \\
0 \\
0 \\
0 \\
0 \\
0 \\
0 \\
0 \\
0 \\
0 \\
0
\end{tabular}

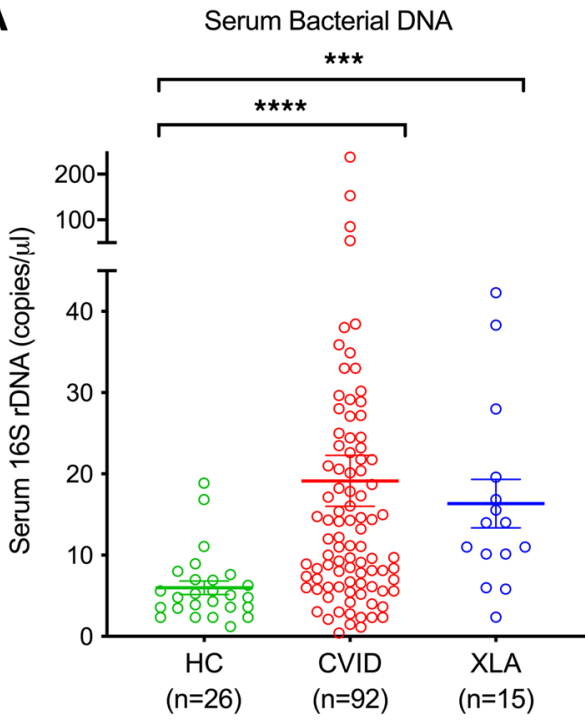

C
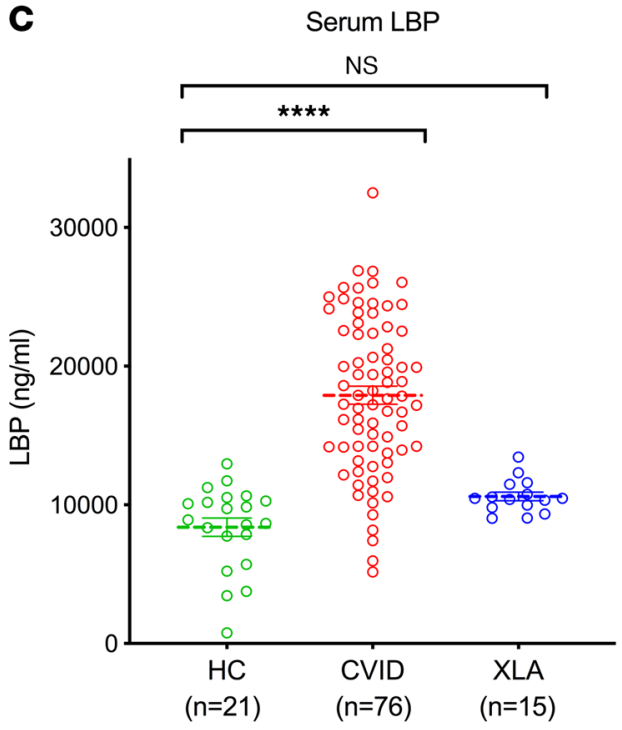

B

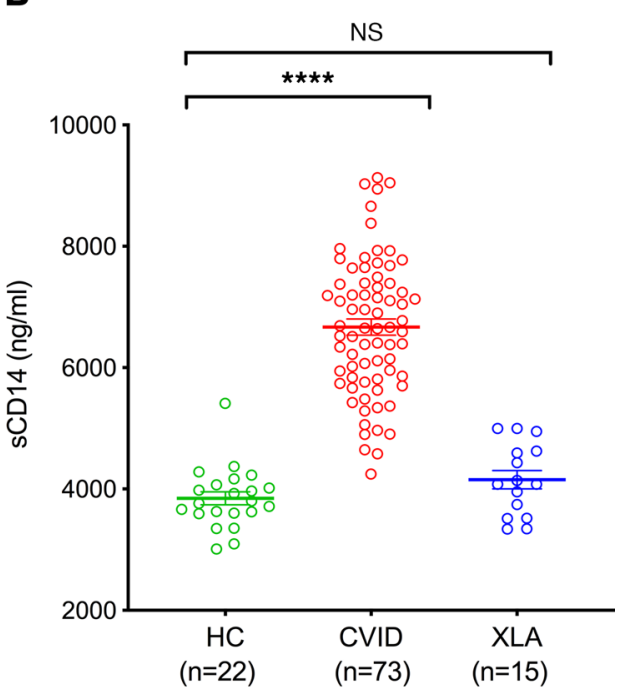

Figure 1. Serum circulating bacterial DNA, SCD14, and LBP levels in CVID as compared with XLA and healthy individuals. (A) Serum 165 rDNA, (B) serum sCD14, and (C) serum LBP levels in healthy controls (HCs), patients with CVID, and patients with XLA. Kruskal-Wallis test revealed significant differences between groups for bacterial 165 rDNA $(P<0.0001)$; 1-way ANOVA revealed significant differences between groups for sCD14 $(P<0.0001)$ and LBP $(P$ $<0.0001)$. The data are expressed as the mean \pm SEM. ${ }^{* * *} P<0.001,{ }^{* * * *} P<0.0001$ by Kruskal-Wallis with Dunn's multiple-comparison post hoc test (A) and 1-way ANOVA with Tukey's post hoc test (B and C). NS, not significant.

memory B cells (defined as $<2 \%$ of total $\mathrm{CD} 19^{+} \mathrm{B}$ cells) (41) had significantly higher serum bacterial $16 \mathrm{~S}$ rDNA levels as compared with those with higher numbers of these cells (mean 19.27 copies/L, range $0.4-85$ copies $/ \mathrm{L}$ vs. mean 10.24 copies $/ \mathrm{L}$, range $1.14-34.9$ copies $/ \mathrm{L}$, respectively, $P=0.0008$, Figure 4D). Overall, there was a negative correlation between percentages of isotype-switched memory B cells and circulating bacterial DNA levels (Supplemental Figure 4). There was no correlation between bacterial $16 \mathrm{~S}$ rDNA levels and circulating $\mathrm{CD}^{+}, \mathrm{CD}^{+}$, or $\mathrm{CD}^{+} \mathrm{T}$ cell counts (not shown). In our cohort, we also did not identify serum bacterial DNA differences among the subset of 16 patients with known monogenic defects (TNFRSF13B, $n=7$; CTLA4, $n=3 ;$ STAT3 gain of function, $n=2 ;$ IKZF1, KMT2D, $N F K B 1, P I K 3 C A, n=1$ each; Supplemental Figure 5).

Bacterial DNA as an immune stimulant for IFN- $\gamma$ production in CVID. We previously showed that marked upregulation of IFN-related pathways distinguished patients with CVID with inflammatory conditions from those without and that patients with inflammatory CVID had detectable serum IFN- $\gamma(10,11)$. This was 
A

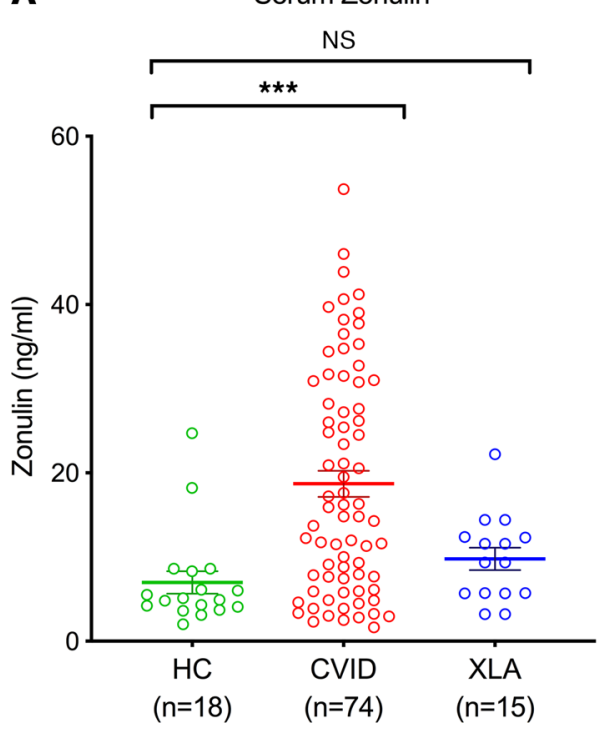

B

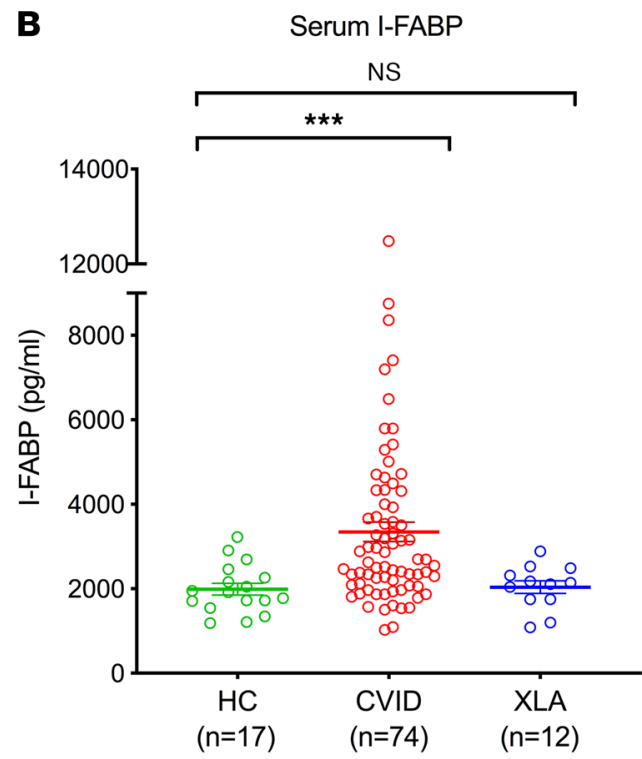

Figure 2. Mucosal epithelial barrier dysfunction in CVID. (A) Serum zonulin and (B) I-FABP levels in healthy controls $(H C s)$, patients with CVID, and patients with XLA. Kruskal-Wallis test revealed significant differences between groups for zonulin $(P=0.0003)$ and I-FABP $(P=0.0002)$. The data are expressed as the mean $\pm \mathrm{SEM} .{ }^{* * *} P<0.001$ by Kruskal-Wallis with Dunn's multiple-comparison post hoc test. NS, not significant.

confirmed here: serum IFN- $\gamma$ levels were significantly elevated in these individuals with CVID when compared with other patients with CVID, patients with XLA, and healthy controls (mean $228.6 \mathrm{pg} / \mathrm{mL}, 118.6$ $\mathrm{pg} / \mathrm{mL}, 182.8 \mathrm{pg} / \mathrm{mL}$, and $109.5 \mathrm{pg} / \mathrm{mL}$, respectively, $P<0.0001$, Figure $5 \mathrm{~A}$ ). IFN- $\gamma$ levels were also modestly elevated in XLA, but this was not significant when compared with healthy controls (Figure 5A).

Next, we sought to determine whether circulating bacterial DNA might be bioactive and induce significant IFN- $\gamma$ secretion in CVID. In vivo, we found that patients with CVID with high serum bacterial DNA (defined as $>90$ th percentile of healthy controls) had significantly higher same-day serum IFN- $\gamma$ levels compared with those with lower serum 16S rDNA (mean IFN- $\gamma 242.3 \mathrm{pg} / \mathrm{mL}$ vs. $169.7 \mathrm{pg} / \mathrm{mL}$, respectively, $P=0.0258$, Figure 5B). To further investigate this, we cultured PBMCs isolated from patients with CVID and controls with the $16 \mathrm{~S}$ bacterial DNA standard and measured IFN- $\gamma$ production. Bacterial DNA effectively induced IFN- $\gamma$ production in all study participants, but it induced significantly higher IFN- $\gamma$ secretion in patients with CVID who had inflammatory conditions, as compared with other patients with CVID, patients with XLA, and healthy controls (mean $560.0 \mathrm{pg} / \mathrm{mL}, 197.7 \mathrm{pg} / \mathrm{mL}, 188.5 \mathrm{pg} / \mathrm{mL}$, and $258.6 \mathrm{pg} /$ $\mathrm{mL}$, respectively, $P<0.0001$, Figure $5 \mathrm{C}$ ) in a dose-dependent manner (Supplemental Figure 6). The digestion of bacterial DNA by DNase abrogated the IFN- $\gamma$ response in the PBMC coculture, demonstrating the specificity of this response (Supplemental Figure 7). Separately, we asked whether the presence of human cell-free DNA might enhance or inhibit the observed IFN- $\gamma$ response among patients with CVID in this assay: no changes in IFN- $\gamma$ response were observed (data not shown).

$B$ cell numbers, Bruton tyrosine kinase, and IFN- $\gamma$ production. Given the differential response between CVID and patients with XLA, we next examined the role of B cells in this bacterial-driven response. However, there was no significant correlation between peripheral B cell numbers and serum IFN- $\gamma$ levels in vivo in patients with CVID (Supplemental Figure 8). Similarly, we did not find that the in vitro bacterial DNAstimulated IFN- $\gamma$ response among the very rare patients with CVID who had inflammatory complications with an absence of peripheral B cells was significantly different from those with peripheral B cells (mean IFN- $\gamma: 555.6 \mathrm{pg} / \mathrm{mL}[n=3]$ vs. $563.3 \mathrm{pg} / \mathrm{mL}[n=4]$, respectively). We then investigated whether the loss of Bruton tyrosine kinase (BTK) signals in XLA could lead to the differential IFN- $\gamma$ response to bacterial stimuli between patients with CVID and patients with XLA. To answer this question, we examined the effect of BTK inhibition (42) in PBMC cultures of patients with CVID and inflammatory complications. Indeed, we found that BTK inhibition significantly reduced the IFN- $\gamma$ response to bacterial DNA stimulation in these patients with CVID (mean IFN- $\gamma 476.0 \mathrm{pg} / \mathrm{mL}$ [bacterial DNA stimulation] vs. $83.9 \mathrm{pg} / \mathrm{mL}$ [bacterial DNA stimulation + BTK inhibition], $P<0.0001$, Figure 5D). 


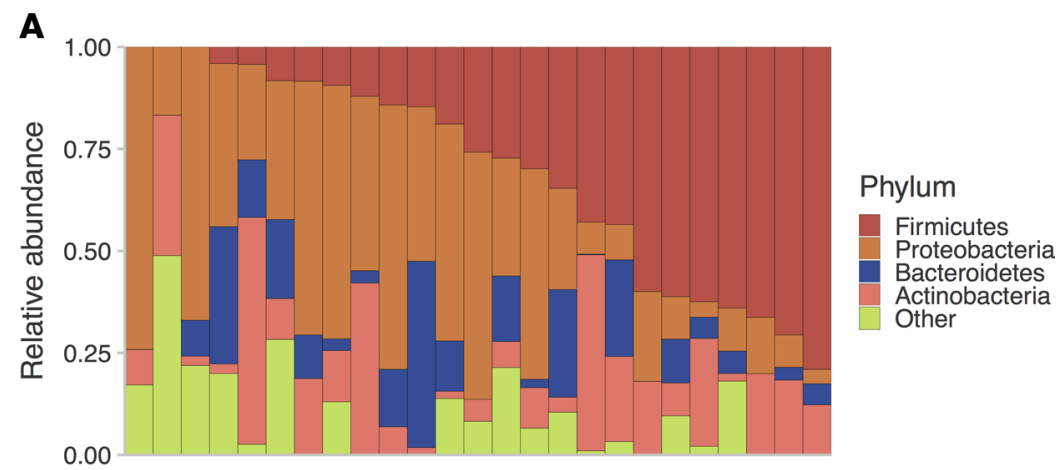

B

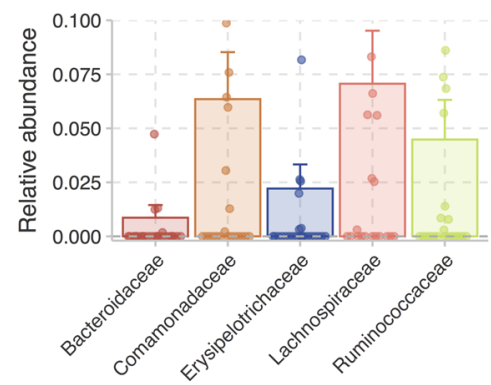

Figure 3. 165 profiling of circulating bacterial DNA in CVID identified genetic materials belonging to gut-associated commensals and an abundance of bacterial families that are normally highly coated by secretory lgA/lgM. (A) Relative abundances of bacterial phyla detected in the sera of patients with CVID $(n=25)$. Each column represents serum from a patient with CVID. (B) Most abundant bacterial families detected in the sera of patients with CVID ( $n$ $=25)$. The data are expressed as the mean relative abundance \pm SEM.

Taken together, these results indicate that circulating bacterial DNA is bioactive and capable of driving systemic immune activation in susceptible hosts and, in particular, the IFN- $\gamma$ signature we previously observed in patients with CVID and inflammatory complications.

\section{Discussion}

The compartmentalization of host and commensals is essential for health, preventing microbial-driven inflammation and translocation. In this study, we demonstrated direct evidence of bacterial translocation from mucosal surfaces into the systemic circulation (16S rDNA) in patients with CVID, as well as XLA, both primary immune defects marked by a profound loss of humoral immunity. Bacterial translocation in CVID has previously been hypothesized, though not proven, based on indirect markers of cellular activation. Perreau et al. reported that bacteria-specific CD $4^{+} \mathrm{T}$ cells from patients with CVID expressed higher levels of PD-1, suggesting chronic bacterial antigen exposure (27). Jorgensen et al. noted soluble CD25 in plasma of patients with CVID with altered gut microbiota (43). However, direct evaluations, such as measuring circulating LPS (a component of Gram-negative bacterial cell wall), have yielded contradictory results in CVID, with detection of LPS/endotoxin in some patients with low serum IgG $(27,28)$; other studies have showed no differences in circulating LPS levels between patients with CVID and controls using the same assay $(29,30)$. Here, in a large cohort of 92 patients with CVID, we also did not detect circulating endotoxin/LPS using 4 commercial assays. Given that 90 out of 92 patients with CVID were on Ig replacement therapy, this finding may reflect the rapid binding of endotoxin/LPS by infused IgG antibody $(27,44,45)$. In this cohort, all patients with replaced IgG had serum IgG levels (mean $999 \mathrm{mg} / \mathrm{dL}$ ) well above a level previously suggested as sufficient for reducing circulating endotoxins ( $490 \mathrm{mg} / \mathrm{dL})(27)$. It is also possible that infused $\mathrm{IgG}$ in patients interferes with the endotoxin/LPS detection assay, which we also demonstrated here in vitro. However, by measuring conserved bacterial $16 \mathrm{~S}$ rDNA in the serum, we were able to circumvent these limitations, and we established the existence of bacterial translocation from the gut to circulation in patients with CVID and in patients with XLA. 
A

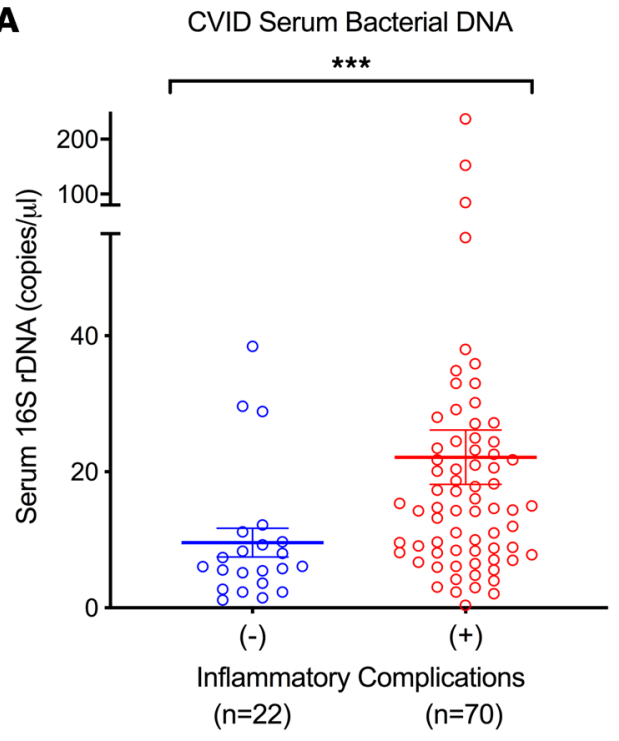

C

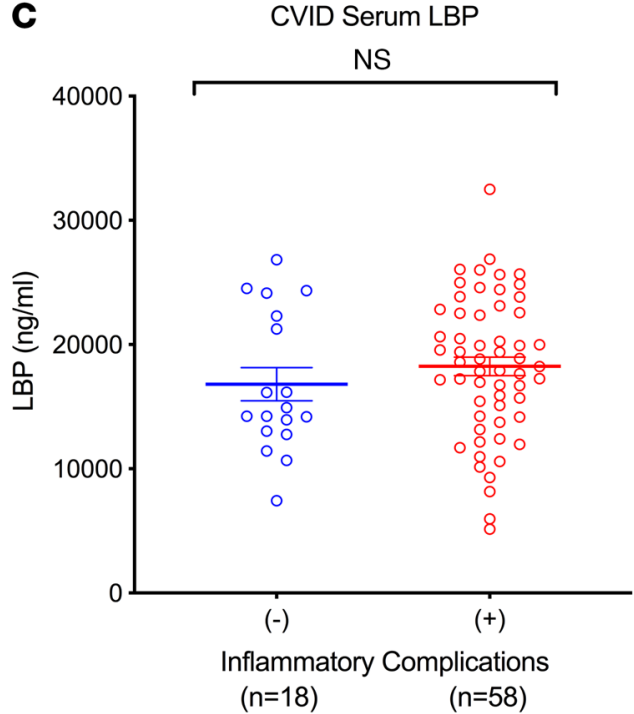

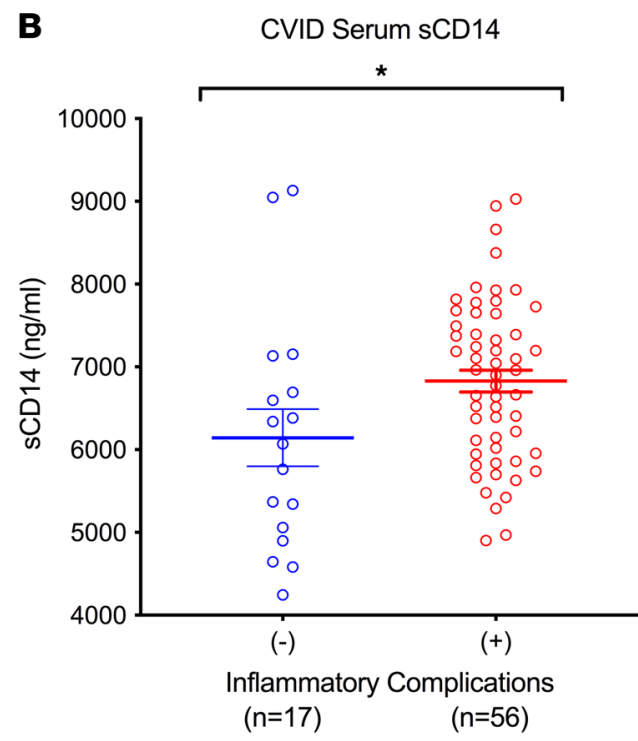

D

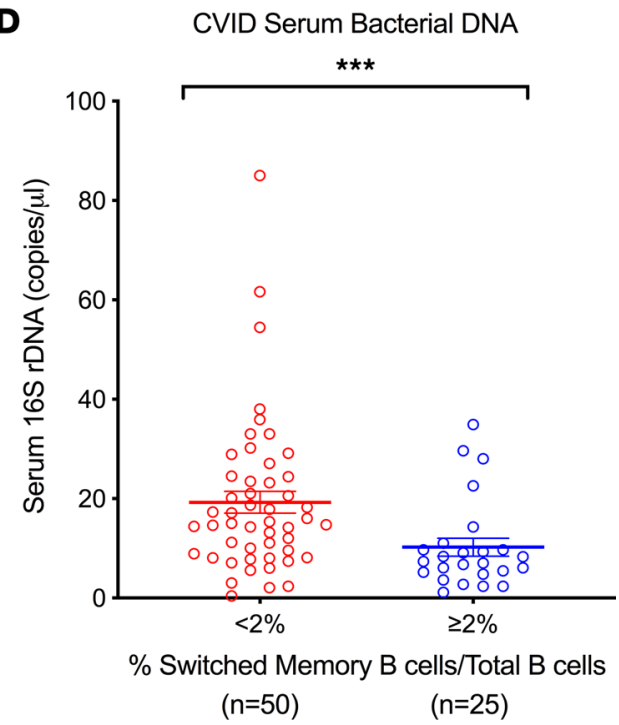

Figure 4. Circulating bacterial DNA is related to inflammatory manifestation in CVID. Comparison of (A) serum 165 rDNA, (B) serum SCD14, and (C) LBP levels in patients with CVID with and without autoimmune/inflammatory complications. Mann-Whitney $U$ test revealed significant differences between groups for bacterial 165 rDNA $(P=0.0007)$. Unpaired $t$ test revealed significant differences between groups for sCD14 $(P=0.027)$. (D) Comparison of serum 165 rDNA levels in patients with CVID with less than $2 \%$ versus $2 \%$ or more of $C D 19{ }^{+} C D 27^{+} \lg M^{-} \mid g D^{-}$switched memory $B$ cells/CD19+ $B$ cells. Mann-Whitney $U$ test revealed significant differences between groups for bacterial 165 rDNA $(P=$ 0.0008). The data are expressed as the mean \pm SEM. ${ }^{*} P<0.05$, ${ }^{* *} P<0.001$ by Mann-Whitney $U$ test $(\mathbf{A}$ and $\mathbf{D})$ and unpaired $t$ test (B). NS, not significant.

In CVID, detection of significantly elevated circulating bacterial DNA was accompanied by elevated measures of systemic immune activation: serum LBP and sCD14. Our results are consistent with prior reports noting increased SCD14 in smaller CVID cohorts $(29,30)$ and in other disease states characterized by the presence of bacterial translocation $(46,47)$. Soluble CD14, in particular, may be released in the setting of exposure to various microbial products, including LPS (Gram-negative bacteria), Gram-positive bacteria components, and CpG DNA $(23-26,48)$. Consistent with these findings, we observed a positive correlation between $\mathrm{sCD} 14$ and serum $16 \mathrm{~S}$ rDNA levels, suggesting that its elevation reflects the translocated bacterial DNA as an immune stimulant in this disorder.

Further evidence of a specific gastrointestinal barrier defect in CVID was identified with elevated serum levels of both zonulin and I-FABP. As known markers of gut epithelial integrity, increased serum zonulin 
A

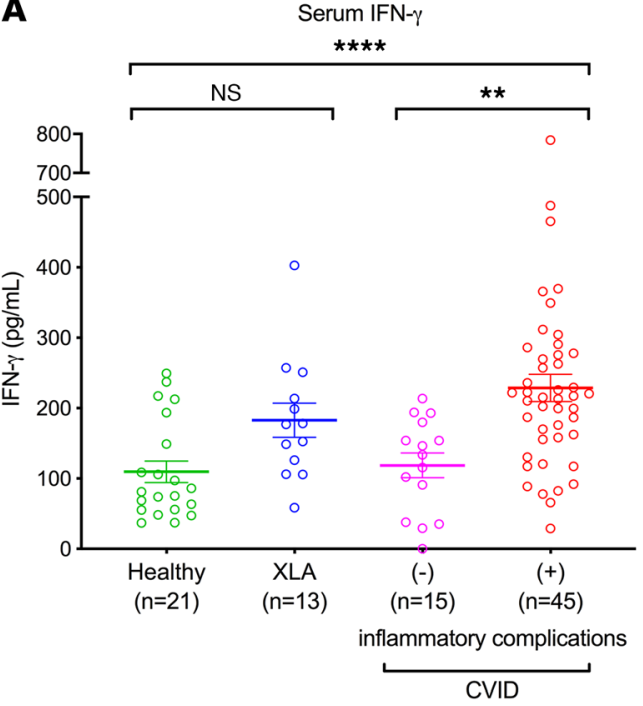

B

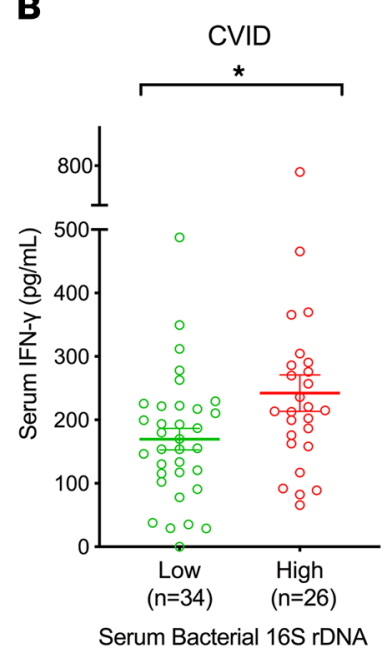

C

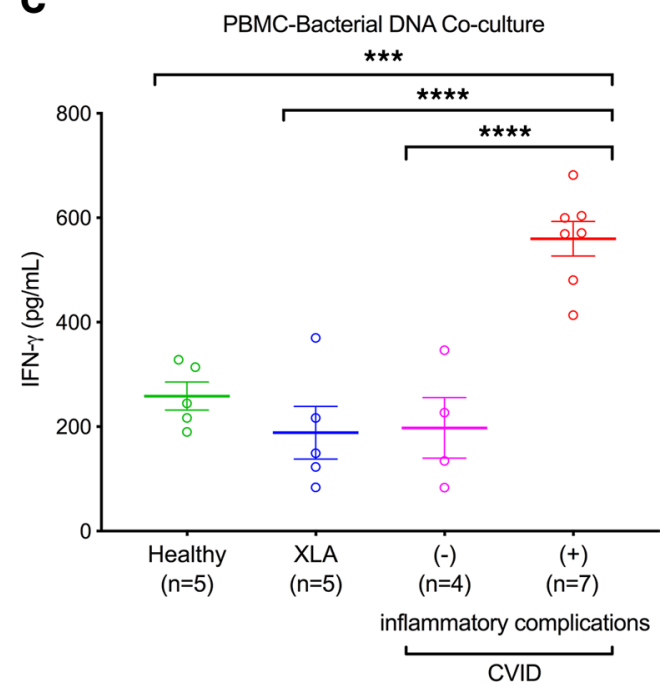

D Bacterial DNA PBMC Co-culture
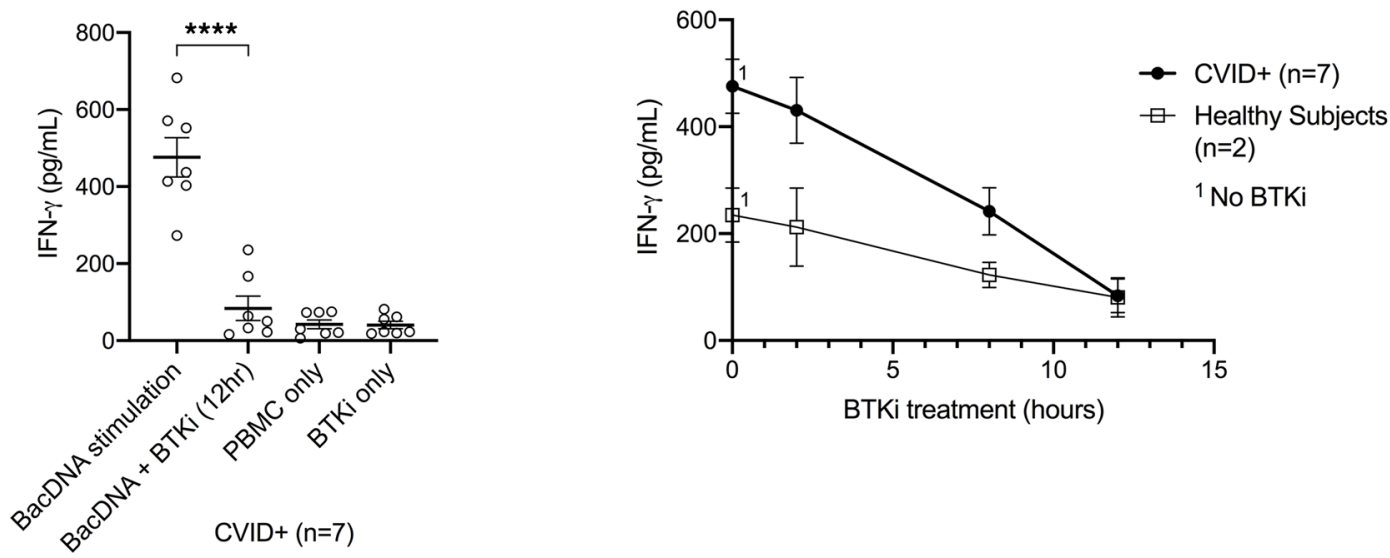

Figure 5. Bacterial DNA is an immune stimulant for IFN- $\gamma$ production in CVID. (A) Serum IFN- $\gamma$ levels in healthy controls (HCs), patients with XLA, and patients with CVID with or without autoimmune/inflammatory complications (CVID+, CVID, respectively). One-way ANOVA revealed significant differences between groups $(P<0.0001)$ (B) Serum IFN- $\gamma$ levels in patients with CVID with low versus high serum bacterial 165 rDNA. High serum bacterial 165 rDNA is defined as levels higher than the 90th percentile of healthy controls. Unpaired $t$ test revealed significant differences between groups $(P=0.0258)$ (C) IFN- $\gamma$ levels in the culture supernatants of PBMCs isolated from HCs and XLA, CVID, and CVID+ patients on day 3 after coculture with bacterial DNA (7.5 pg/mL). One-way ANOVA revealed significant differences between groups $(P<0.0001)$. (D) IFN- $\gamma$ levels in the culture supernatants of PBMCs isolated from CVID+ patients on day 3 after coculture with bacterial DNA $(7.5 \mathrm{pg} / \mathrm{mL})$, with or without a BTK inhibitor (BTKi; PCI 29732, $0.5 \mathrm{nM}$; left panel). Unpaired $t$ test revealed significant differences between groups $(P<0.0001)$. Bacterial DNA-induced IFN- $\gamma$ levels in response to BTKi treatment over time $(\mathrm{PCI} 29732,0.5 \mathrm{nM} ; 0-12$ hours) in PBMCs isolated from CVID+ and healthy individuals (right panel). The data are expressed as the mean $\pm \mathrm{SEM} .{ }^{*} P<0.05,{ }^{* *} P<0.01,{ }^{* * *} P<0.001,{ }^{* * * *} P<0.0001$ by 1 -way ANOVA with Tukey's post hoc test ( $\mathbf{A}$ and $\mathbf{C}$ ) and unpaired $t$ test (B and $\mathbf{D})$. NS, not significant.

and I-FABP have similarly been found in celiac disease, inflammatory bowel disease, HIV, and other chronic inflammatory diseases $(47,49-52)$. While specificity of the current commercial zonulin assay has been a topic of debate $(31,53)$, zonulin levels have been correlated with experimental and clinical parameters of intestinal permeability (51). Our results suggest that the combination of humoral immune defects and intestinal barrier dysfunction underlie the bacterial translocation phenomenon in CVID. However, the resulting inflammatory state in CVID may further promote barrier defects, creating a vicious cycle of bacterial translocation and immune stimulation $(54,55)$.

In addition to being associated with markers of immune activation, we showed that high circulating bacterial DNA levels were strongly associated clinically with the inflammatory CVID phenotype in this large cohort. In a similar manner, bacterial translocation has been implicated in a number of chronic inflammatory states, including HIV infection, cirrhosis, and inflammatory bowel diseases, among others $(47,56,57)$. Our finding extends earlier observations that altered gut microbiota was associated with 
markers of immune activation (sCD14, sCD25) in CVID $(30,43)$. With evidence of mucosal barrier dysfunction (elevated zonulin and I-FABP levels), continuous absorption of various bacterial products, including DNA, would likely activate systemic immune responses through recognition by innate pattern recognition receptors (58) with complex immunological consequences.

Focusing on the IFN signature that characterizes the inflammatory state in CVID (10), we found that patients with high circulating bacterial DNA also had high same-day serum IFN- $\gamma$ in vivo. We further demonstrated bacterial DNA-induced IFN- $\gamma$ secretion in both CVID and control PBMCs in vitro. Our findings are consistent with prior observations on the capability of bacterial DNA to induce host immune responses in experimental models (59-61). These results support the hypothesis that the translocated bacteria DNA is bioactive and capable of driving inflammatory complications in CVID. Interestingly, bacterial DNA induced significantly higher IFN- $\gamma$ secretion in patients with CVID with inflammatory complications in comparison to those without complications and healthy controls, suggesting that their cells were primed to respond. Taken together, these findings indicate that it is not the mere presence of an inflammatory stimulus that determines pathology, but that the host's response to such a stimulus likely plays a determining role.

Patients with CVID with severe reduction of isotype-switched memory B cells had significantly elevated circulating $16 \mathrm{~S}$ rDNA levels. The loss of these cells has previously been associated with reduced IgA, IgG, and specific antibody responses, as well as higher risks for inflammatory complications (41, 62-64). In line with previous observations that IgG replacement alone does not appear to ameliorate most inflammatory complications in CVID $(5,6)$, the translocation of bacterial DNA in these patients with CVID was unaffected by IgG replacement therapy. Taken together, these data indicate that severe defects in the B cell compartment are likely related to defects in host-commensal compartmentalization in CVID. Additionally, while IgG replacement may bind or neutralize some bacterial products (e.g., endotoxin), it appeared insufficient to abolish all facets of bacterial translocation. While loss of secretory antibodies (e.g., intestinal IgA) may play a determining role, serum IgA was very low or absent in patients in this study because of their diagnosis.

Interestingly, although elevated circulating bacterial DNA was observed in CVID and in patients with XLA, there was a notable differential host response to microbial stimulus between these 2 groups, with lack of prominent systemic immune activation in XLA (no significant elevations of LBP or sCD14). This finding is consistent with the clinical phenotype of XLA, in that these patients generally lack the typical inflammatory complications seen in CVID (65). XLA results from a B cell differentiation arrest due to mutations in the BTK gene $(66,67)$, but the additional loss of functional BTK in monocytes, macrophages, neutrophils, and DCs in these patients could blunt or eliminate some of the inflammatory responses. Since BTK is involved in multiple pattern recognition receptor signaling pathways (e.g., TLRs) $(68,69)$, the absence of BTK may also dampen the immune response to translocated microbes/microbial products. Indeed, bacterial DNA-induced cytokine production has been shown to be impaired in PBMCs derived from patients with XLA (69). Here, we found a differential bacterial DNA-induced IFN- $\gamma$ response between patients with CVID and patients with XLA, but this did not appear to be a function of B cells alone because there was no significant correlation between peripheral B cell numbers and serum IFN- $\gamma$ levels in vivo among patients with CVID. Given that BTK inhibition resulted in reduced IFN- $\gamma$ responses to bacterial DNA stimuli among patients with CVID and inflammatory complications in vitro, we suggest that BTK is a host modifier in immune responses to microbial translocation. It is also possible that a more intact gut barrier integrity in XLA, as suggested by the relatively normal zonulin and I-FABP levels here, contribute to this differential outcome. Further investigation of the molecular and cellular basis of these differential immune responses in CVID and XLA could be revealing and potentially of therapeutic importance.

By performing 16S rRNA gene profiling, we identified circulating bacterial DNA from a diverse range of commensals, rather than select bacterial species, in patients with CVID. Even though the gut is almost certainly the predominant source of bacterial translocation, we cannot exclude this occurrence from other mucosal sites (e.g., respiratory tract). In the CVID sera, we identified an abundance of Lachnospiraceae, Ruminococcaceae, Erysipelotrichaceae, and Bacteroidaceae DNA - all gut commensal families with members previously reported to be well-coated by secretory IgA in healthy humans (37-39). We also identified an abundance of Comamonadaceae DNA, which was previously found to be highly coated with IgM in people with selective IgA deficiency (40). Jorgensen et al. found an increased abundance of the genera Roseburia (part of the Lachnospiraceae family) and Bacilli (Bacillaceae) in the gut of patients with CVID relative to healthy individuals (43); consistent with this prior finding, we observed that Roseburia (Lachnospiraceae) and Bacilli (Bacillaceae) DNA were highly abundant in CVID sera. In contrast, our serum analysis did not detect 
bacteria DNA from the family Enterobacteriaceae, which was reported to be relatively increased in the gut of patients with CVID compared with healthy controls (70). Lastly, through transkingdom network analysis of the duodenal microbiome in patients with CVID with or without enteropathy, Acinetobacter baumannii has previously been proposed to be a candidate inciting organism in patients with CVID with enteropathy (71); the DNA of this species was, however, not detected in the sera of patients with CVID in this report regardless of the presence or absence of enteropathy.

There are limitations to this study. We detected elevated circulating bacterial product (16S rDNA of various gut taxa) in patients with CVID in the absence of overt bacteremia (i.e., demonstrated growth of intact bacteria through conventional blood cultures), but the lack of direct access to mesenteric vein/lymph nodes in our human study prevented us from more conclusively determining whether there was also the translocation of some intact bacteria through the gut epithelium in CVID. In addition, although bacterial 16S rDNA has been used to detect bacterial translocation in other fields, its precise form(s) in the circulation remain to be fully elucidated. Future dedicated structural-based studies on the biochemical forms of the translocated bacterial DNA and whether it may be bound to additional bacterial debris may also be revealing.

In summary, we showed that bacterial translocation in patients with profound primary antibody defects leads to circulating bioactive bacterial DNA capable of immune activation and is associated with IFN- $\gamma$ linked inflammatory manifestations in CVID. These data established a mechanism for immune activation in CVID and identified bacterial DNA translocation, barrier dysfunction, and the ensuing host response as interventional targets to modify the clinical outcomes of CVID. Future studies of host-commensal immune regulation in this and other human primary immunodeficiency disorders should improve our understanding of this complex relationship and therapeutic approaches.

\section{Methods}

Patients and serum. All participants recruited for this study were patients at the Mount Sinai Clinical Immunology Faculty Practice. Serum from patients with CVID $(1,2)$, patients with XLA (1), and healthy controls were drawn into $6 \mathrm{~mL}$ gold top rubber-sealed sterile Vacutainer SST II tubes (BD Diagnostics). Baseline patient characteristics are summarized in Table 1. In the CVID cohort, autoimmunity/inflammatory complications included lymphoid hyperplasia/splenomegaly $(n=52)$, hematologic autoimmunity (autoimmune hemolytic anemia and/or immune thrombocytopenic purpura, $n=38$ ), chronic lung disease (interstitial lung disease and/or granulomatous lung disease, $n=35$ ), enteropathy ( $n=33$ ), and granulomas $(n=26)$. The tubes were maintained in the upright position until the serum was aseptically removed in a sterile hood for bacterial and other assays.

Quantitative PCR for bacterial $16 S$ rDNA. Quantitative PCR was performed blinded for clinical characteristics. The amplification reaction mixture was composed of $10 \mu \mathrm{L}$ of LightCycler $480 \mathrm{SYBR}$ Green (Roche), $1 \mu \mathrm{L}$ of $16 \mathrm{~S}$ bacterial rDNA forward primer (5'-AAC AGG ATT AGA TAC CCT GGT AG-3', $1 \mu \mathrm{L}$ of reverse primer (5'-GGT TCT KCG CGT TGC WTC-3') (72), $1 \mu \mathrm{L}$ of dsDNAase (Thermo Fisher Scientific), $1 \mu \mathrm{L}$ of $10 \times$ dsDNAase buffer (Thermo Fisher Scientific), and $5 \mu \mathrm{L}$ of SYBR Green reaction mix (Roche). Then, $19 \mu \mathrm{L}$ of the reaction mixture was added to each well of a 384-qPCR plate, followed by $1 \mu \mathrm{L}$ of DNA isolated from $200 \mu \mathrm{L}$ of serum (DNeasy Blood and Tissue Kit, Qiagen) or the E. coli bacterial DNA standard. The DNA was amplified in triplicate, and mean values were calculated. The reaction conditions for amplification of DNA were $95^{\circ} \mathrm{C}$ for 10 minutes, followed by 45 cycles at $95^{\circ} \mathrm{C}$ for 10 seconds, $60^{\circ} \mathrm{C}$ for 20 seconds, and $72^{\circ} \mathrm{C}$ for 5 seconds. The bacterial DNA standard was prepared from E. coli-competent cells (Thermo Fisher Scientific) using DNeasy Blood and Tissue Kit (Qiagen) and concentrations were measured by NanoDrop (Thermo Fisher Scientific). Using the E. coli genome length (4,700,000 bp), the number of E. coli DNA copies was calculated (https://cels.uri.edu/gsc/cndna.html) and serial dilutions of this standard were used to define DNA copy numbers in a standard curve.

Serum LBP, sCD14, zonulin, I-FABP, and IFN- $\gamma$ assays. Concentrations of sCD14, LBP, zonulin, and I-FABP were determined in serum (with dilutions of 1:2500, 1:1000, 1:10, 1:10, respectively) using separate immunoassays (R\&D Systems). Serum IFN- $\gamma$, diluted 1:2, from patients not on a systemic immunosuppressant was determined using a human IFN- $\gamma$ ELISA (R\&D Systems). FLUOstar Omega multimode microplate reader (BMG Labtech) was employed. Representative data from duplicate experiments were presented.

Endotoxin/LPS assays. The endotoxin detection assays used were EndoLISA (Hyglos), Pierce LAL Chromogenic Endotoxin Quantitation kit (Thermo Fisher Scientific), Limulus Amebocyte Lysate Chromogenic Endpoint Assay (Hycult Biotech), and ToxinSensor (GenScript). To examine potential 
interference by Ig, bacterial endotoxin (Invivogen) was coated on ELISA plates (Thermo Fisher Scientific); subsequently, 0.003 to $11.4 \mathrm{mg} / \mathrm{mL}$ of a commercial polyclonal Ig was added and left to bind for 2 hours at room temperature. Detection of endotoxin-bound commercial Ig was carried out with HRP-conjugated anti-human IgG antibody (Southern Biotech).

Flow cytometry. Four-color flow cytometry with LSRII cytometer and FacsDIVA software (BD Biosciences) was performed on patient PBMCs using anti-human mAbs CD19 PC5 (Beckman Coulter, IM2643U), CD27 FITC (Dako, F7178), IgM APC (Jackson ImmunoResearch, 309605095), and IgD PE (BD Pharmingen, 555779). Analysis was performed with FlowJo software.

DNA extraction, $16 S \mathrm{rDNA}$ amplification, and multiplex sequencing. DNA was extracted from collected 25 CVID serum samples following handling guidelines for microbiome studies as previously described (73). Briefly, collected serum samples were introduced into a biosafety cabinet that was decontaminated prior to introduction of the samples by UV treatment for 1 hour and subsequently decontaminated with $5 \%$ bleach. Personnel handling the samples were wearing isolation gowns, clean gloves that were sprayed beforehand with 5\% bleach and 70\% ethanol, and a face mask. DNA was extracted using the DNeasy UltraClean Microbial Kit (Qiagen), including 12 blank-extraction controls. Amplicon preparation and sequencing were performed as previously described (74). Briefly, bacterial 16S rDNA PCR, including 25 no-template PCR controls, was set up in a separate PCR workstation using dual-indexed primers. PCR reactions contained $1 \mu \mathrm{M}$ for each primer, $4 \mathrm{ng}$ DNA, and Phusion Flash High-Fidelity PCR Master Mix (Thermo Fisher Scientific). Reactions were held at $98^{\circ} \mathrm{C}$ for 30 seconds, proceeding to 50 cycles at $98^{\circ} \mathrm{C}$ for 10 seconds, $45^{\circ} \mathrm{C}$ for 30 seconds, and $72^{\circ} \mathrm{C}$ for 30 seconds and a final extension of 2 minutes at $72^{\circ} \mathrm{C}$. Amplicons were evaluated by gel electrophoresis. The sequencing library was prepared by combining equivolume amounts of each amplicon, size-selected and concentrated using AMPure XP beads $(0.8 \times$, Beckman Coulter). Library concentration was quantified by Qubit (Thermo Fisher Scientific) and $\mathrm{qPCR}$, mixed with $20 \% \mathrm{PhiX}$, diluted to $4 \mathrm{pM}$, and subjected to paired-end sequencing (Reagent Kit V2, $2 \times 150 \mathrm{bp}$ ) on an Illumina MiSeq sequencer.

Resulting FASTQ files were analyzed using the QIIME2 version 2019.10 (75) and the DADA2 (76) denoised-paired plugin with a truncation length of $150 \mathrm{bp}$ and $145 \mathrm{bp}$ for the forward and reverse read, respectively. Amplicon sequence variants (ASVs) were classified using the Scikit-Learn plugin (77) using the gg-13-8-99-515-806-nb classifier. Resulting ASV tables were filtered using a minimum depth of 1000 and a minimal ASV frequency of 30, and any ASVs observed in the blank extraction and nontemplate PCR controls were filtered out. Taxonomic plots were generated from the filtered ASV tables using R/ggplot2 (78).

Bacterial DNA and PBMC coculture. Bacterial DNA was prepared from E. coli-competent cells (Thermo Fisher Scientific) using DNeasy Blood and Tissue Kit (Qiagen). PBMCs freshly isolated from each study participant $\left(5 \times 10^{5}\right.$ cells $)$ were cocultured with $E$. coli bacterial DNA $\left(0\right.$ to $3 \times 10^{4}$ DNA copies $[7.5 \mathrm{pg} / \mathrm{mL}])$. Coculture supernatants were collected and analyzed on day 3 . IFN- $\gamma$ levels in the supernatants were measured using a human IFN- $\gamma$ ELISA (R\&D Systems) and the FLUOstar Omega multimode microplate reader (BMG Labtech). For bacterial DNA digestion, E. coli bacterial DNA was incubated with DNase I (Thermo Fisher Scientific) at $37^{\circ} \mathrm{C}$ for 15 minutes. For BTK inhibition, PCI 29732 (final concentration $0.5 \mathrm{nM}$, Tocris Bioscience) (42) was added to PBMC cultures at 2, 8, and 12 hours prior to the addition of $E$. coli bacterial DNA $\left(3 \times 10^{4}\right.$ copies, $\left.7.5 \mathrm{pg} / \mathrm{mL}\right)$. To examine potential interference by human cell-free DNA, an equivalent concentration $(7.5 \mathrm{pg} / \mathrm{mL})$ of human genomic DNA (Promega) was added to the bacterial DNA and PBMC coculture, and IFN- $\gamma$ output was measured as described previously.

Statistics. Statistical differences for 2-group comparisons were analyzed using an 2-tailed, unpaired $t$ test for normally distributed data and a Mann-Whitney $U$ test for nonnormal data. Multiple group comparisons were analyzed using 1-way ANOVA with Tukey's multiple-comparison test for normally distributed data and Kruskal-Wallis test with Dunn's multiple-comparison test for nonnormal data. Bivariate correlations between variables were calculated using Spearman's test. All reported $P$ values were 2 -sided, and $P$ values less than 0.05 were considered significant. All calculations were performed using GraphPad Prism software.

Study approval. This study was approved by the IRB of the Icahn School of Medicine at Mount Sinai and was carried out in accordance with the Code of Ethics of the World Medical Association (Declaration of Helsinki). Written informed consent was received from participants prior to inclusion in the study. 


\section{Author contributions}

$\mathrm{HH}$ and $\mathrm{CC}$ conceived the project, designed and performed research, analyzed and discussed data, prepared the figures, and wrote the manuscript. LR and GB performed experiments and analyzed and discussed data. AE performed experiments and discussed data. All authors contributed to critical revision of the manuscript and approved the final draft.

\section{Acknowledgments}

We thank the patients and their families for their participation in this research as well as the nursing and support staff for sharing in the care of these patients.

This work was supported by the NIH (AI-061093, AI-086037, AI-48693) and the David S. Gottesman Immunology Chair.

Address correspondence to: Charlotte Cunningham-Rundles, 1425 Madison Avenue, New York, New York 10029, USA. Phone: 212.659.9268; Email: charlotte.cunningham-rundles@mssm.edu.

AE's present address is: Department of Pharmaceutical and Biomedical Sciences, California Northstate University College of Pharmacy, Elk Grove, California, USA.

1. Tangye SG, et al. Human inborn errors of immunity: 2019 update on the classification from the International Union of Immunological Societies Expert Committee. J Clin Immunol. 2020;40(1):24-64.

2. Bonilla FA, et al. International consensus document (ICON): common variable immunodeficiency disorders. $J$ Allergy Clin Immunol Pract. 2016;4(1):38-59.

3. Seidel MG, et al. The European Society for Immunodeficiencies (ESID) registry working definitions for the clinical diagnosis of inborn errors of immunity. J Allergy Clin Immunol Pract. 2019;7(6):1763-1770.

4. Chapel H, et al. Common variable immunodeficiency disorders: division into distinct clinical phenotypes. Blood. 2008;112(2):277-286

5. Resnick ES, et al. Morbidity and mortality in common variable immune deficiency over 4 decades. Blood. 2012;119(7):1650-1657.

6. Cunningham-Rundles C, Bodian C. Common variable immunodeficiency: clinical and immunological features of 248 patients. Clin Immunol. 1999;92(1):34-48.

7. Ho HE, Cunningham-Rundles C. Non-infectious complications of common variable immunodeficiency: updated clinical spectrum, sequelae, and insights to pathogenesis. Front Immunol. 2020;11:149.

8. Maffucci P, et al. Genetic diagnosis using whole exome sequencing in common variable immunodeficiency. Front Immunol. 2016;7:220

9. Abolhassani H, et al. Current genetic landscape in common variable immune deficiency. Blood. 2020;135(9):656-667.

10. Park J, et al. Interferon signature in the blood in inflammatory common variable immune deficiency. PLoS One. 2013;8(9):e74893.

11. Cols M, et al. Expansion of inflammatory innate lymphoid cells in patients with common variable immune deficiency. $J$ Allergy Clin Immunol. 2016;137(4):1206-1215.

12. Panda SK, Colonna M. Innate lymphoid cells in mucosal immunity. Front Immunol. 2019;10:861.

13. Sterlin D, et al. The antibody/microbiota interface in health and disease. Mucosal Immunol. 2020;13(1):3-11.

14. Wilmore JR, et al. Commensal microbes induce serum IgA responses that protect against polymicrobial sepsis. Cell Host Microbe. 2018;23(3):302-311.

15. Bunker JJ, et al. Innate and adaptive humoral responses coat distinct commensal bacteria with immunoglobulin A. Immunity. 2015;43(3):541-553.

16. Bunker JJ, et al. Natural polyreactive IgA antibodies coat the intestinal microbiota. Science. 2017;358(6361):eaan6619.

17. Bioley G, et al. Plasma-derived polyreactive secretory-like IgA and IgM opsonizing Salmonella enterica typhimurium reduces invasion and gut tissue inflammation through agglutination. Front Immunol. 2017;8:1043.

18. Zeng MY, et al. Gut microbiota-induced immunoglobulin G controls systemic infection by symbiotic bacteria and pathogens. Immunity. 2016;44(3):647-658.

19. Christmann BS, et al. Human seroreactivity to gut microbiota antigens. J Allergy Clin Immunol. 2015;136(5):1378-1386.

20. Fadlallah J, et al. Synergistic convergence of microbiota-specific systemic IgG and secretory IgA. J Allergy Clin Immunol. 2019;143(4):1575-1585.

21. Brenchley JM, et al. Microbial translocation is a cause of systemic immune activation in chronic HIV infection. Nat Med. 2006;12(12):1365-1371.

22. Sartor RB. Microbial influences in inflammatory bowel diseases. Gastroenterology. 2008;134(2):577-594.

23. Marcos V, et al. Expression, regulation and clinical significance of soluble and membrane CD14 receptors in pediatric inflammatory lung diseases. Respir Res. 2010;11:32.

24. Kol A, et al. Cutting edge: heat shock protein (HSP) 60 activates the innate immune response: CD14 is an essential receptor for HSP60 activation of mononuclear cells. J Immunol. 2000;164(1):13-17.

25. Pugin J, et al. CD14 is a pattern recognition receptor. Immunity. 1994;1(6):509-516.

26. Pugin J, et al. Lipopolysaccharide activation of human endothelial and epithelial cells is mediated by lipopolysaccharide-bind- 
ing protein and soluble CD14. Proc Natl Acad Sci U S A. 1993;90(7):2744-2748.

27. Perreau M, et al. Exhaustion of bacteria-specific CD4 T cells and microbial translocation in common variable immunodeficiency disorders. J Exp Med. 2014;211(10):2033-2045.

28. Romberg N, et al. Patients with common variable immunodeficiency with autoimmune cytopenias exhibit hyperplastic yet inefficient germinal center responses. J Allergy Clin Immunol. 2019;143(1):258-265.

29. Barbosa RR, et al. Monocyte activation is a feature of common variable immunodeficiency irrespective of plasma lipopolysaccharide levels. Clin Exp Immunol. 2012;169(3):263-272.

30. Litzman J, et al. Chronic immune activation in common variable immunodeficiency (CVID) is associated with elevated serum levels of soluble CD14 and CD25 but not endotoxaemia. Clin Exp Immunol. 2012;170(3):321-332.

31. Wang W, et al. Human zonulin, a potential modulator of intestinal tight junctions. J Cell Sci. 2000;113 Pt 24:4435-4440.

32. El Asmar R, et al. Host-dependent zonulin secretion causes the impairment of the small intestine barrier function after bacterial exposure. Gastroenterology. 2002;123(5):1607-1615.

33. Sturgeon C, Fasano A. Zonulin, a regulator of epithelial and endothelial barrier functions, and its involvement in chronic inflammatory diseases. Tissue Barriers. 2016;4(4):e1251384.

34. Gollin G, et al. Intestinal fatty acid binding protein in serum and urine reflects early ischemic injury to the small bowel. Surgery. 1993;113(5):545-551.

35. Kanda T, et al. Intestinal fatty acid-binding protein as a sensitive marker of intestinal ischemia. Dig Dis Sci. 1992;37(9):1362-1367.

36. Macpherson AJ, Harris NL. Interactions between commensal intestinal bacteria and the immune system. Nat Rev Immunol. 2004;4(6):478-485.

37. Magri G, et al. Human secretory IgM emerges from plasma cells clonally related to gut memory B cells and targets highly diverse commensals. Immunity. 2017;47(1):118-134.

38. Planer JD, et al. Development of the gut microbiota and mucosal IgA responses in twins and gnotobiotic mice. Nature. 2016;534(7606):263-266.

39. Dzidic M, et al. Aberrant IgA responses to the gut microbiota during infancy precede asthma and allergy development. $J$ Allergy Clin Immunol. 2017;139(3):1017-1025.

40. Catanzaro JR, et al. IgA-deficient humans exhibit gut microbiota dysbiosis despite secretion of compensatory IgM. Sci Rep. 2019;9(1):13574.

41. Wehr C, et al. The EUROclass trial: defining subgroups in common variable immunodeficiency. Blood. 2008;111(1):77-85.

42. Honigberg LA, et al. The Bruton tyrosine kinase inhibitor PCI-32765 blocks B-cell activation and is efficacious in models of autoimmune disease and B-cell malignancy. Proc Natl Acad Sci U S A. 2010;107(29):13075-13080.

43. Jorgensen SF, et al. Altered gut microbiota profile in common variable immunodeficiency associates with levels of lipopolysaccharide and markers of systemic immune activation. Mucosal Immunol. 2016;9(6):1455-1465.

44. Angus DC, et al. Epidemiology of severe sepsis in the United States: analysis of incidence, outcome, and associated costs of care. Crit Care Med. 2001;29(7):1303-1310.

45. Shankar-Hari M, et al. Bench-to-bedside review: immunoglobulin therapy for sepsis - biological plausibility from a critical care perspective. Crit Care. 2012;16(2):206.

46. Albillos A, et al. Increased lipopolysaccharide binding protein in cirrhotic patients with marked immune and hemodynamic derangement. Hepatology. 2003;37(1):208-217.

47. Hunt PW, et al. Gut epithelial barrier dysfunction and innate immune activation predict mortality in treated HIV infection. J Infect Dis. 2014;210(8):1228-1238.

48. Burgmann H, et al. Increased serum concentration of soluble CD14 is a prognostic marker in gram-positive sepsis. Clin Immunol Immunopathol. 1996;80(3 pt 1):307-310.

49. Fasano A, et al. Zonulin, a newly discovered modulator of intestinal permeability, and its expression in coeliac disease. Lancet 2000;355(9214):1518-1519.

50. Drago S, et al. Gliadin, zonulin and gut permeability: effects on celiac and non-celiac intestinal mucosa and intestinal cell lines. Scand J Gastroenterol. 2006;41(4):408-419.

51. Fasano A. Intestinal permeability and its regulation by zonulin: diagnostic and therapeutic implications. Clin Gastroenterol Hepatol. 2012;10(10):1096-1100.

52. Wiercinska-Drapalo A, et al. Intestinal fatty acid binding protein (I-FABP) as a possible biomarker of ileitis in patients with ulcerative colitis. Regul Pept. 2008;147(1-3):25-28.

53. Ajamian M, et al. Serum zonulin as a marker of intestinal mucosal barrier function: may not be what it seems. PLoS One. 2019;14(1):e0210728.

54. Bruewer $\mathrm{M}$, et al. Interferon-gamma induces internalization of epithelial tight junction proteins via a macropinocytosis-like process. FASEB J. 2005;19(8):923-933.

55. Hietbrink F, et al. Systemic inflammation increases intestinal permeability during experimental human endotoxemia. Shock. 2009;32(4):374-378.

56. Giannelli V, et al. Microbiota and the gut-liver axis: bacterial translocation, inflammation and infection in cirrhosis. World J Gastroenterol. 2014;20(45):16795-16810.

57. Vrakas S, et al. Intestinal bacteria composition and translocation of bacteria in inflammatory bowel disease. PLoS One. 2017;12(1):e0170034.

58. Takeuchi O, Akira S. Pattern recognition receptors and inflammation. Cell. 2010;140(6):805-820.

59. Klinman DM, et al. CpG motifs present in bacteria DNA rapidly induce lymphocytes to secrete interleukin 6 , interleukin 12 , and interferon gamma. Proc Natl Acad Sci US A. 1996;93(7):2879-2883.

60. Chace JH, et al. Bacterial DNA-induced NK cell IFN-gamma production is dependent on macrophage secretion of IL-12. Clin Immunol Immunopathol. 1997;84(2):185-193.

61. Bou Ghanem EN, et al. Multiple mechanisms contribute to the robust rapid gamma interferon response by CD8+ T cells during Listeria monocytogenes infection. Infect Immun. 2009;77(4):1492-1501.

62. Ko J, et al. Immune competence and switched memory B cells in common variable immunodeficiency. Clin Immunol. 
2005; 116(1):37-41.

63. Warnatz K, et al. Severe deficiency of switched memory B cells (CD27(+) $\operatorname{IgM}(-) \operatorname{IgD}(-))$ in subgroups of patients with common variable immunodeficiency: a new approach to classify a heterogeneous disease. Blood. 2002;99(5):1544-1551.

64. Sanchez-Ramon S, et al. Memory B cells in common variable immunodeficiency: clinical associations and sex differences. Clin Immunol. 2008;128(3):314-321.

65. Howard V, et al. The health status and quality of life of adults with X-linked agammaglobulinemia. Clin Immunol. 2006;118(2-3):201-208

66. Vetrie D, et al. The gene involved in X-linked agammaglobulinaemia is a member of the src family of protein-tyrosine kinases Nature. 1993;361(6409):226-233.

67. Tsukada S, et al. Deficient expression of a B cell cytoplasmic tyrosine kinase in human X-linked agammaglobulinemia. Cell. 1993;72(2):279-290.

68. Schmidt NW, et al. Bruton's tyrosine kinase is required for TLR-induced IL-10 production. J Immunol. 2006;177(10):7203-7210.

69. Doyle SL, et al. Signaling by Toll-like receptors 8 and 9 requires Bruton's tyrosine kinase. J Biol Chem. 2007;282(51):36953-36960.

70. Fiedorova $\mathrm{K}$, et al. Bacterial but not fungal gut microbiota alterations are associated with common variable immunodeficiency (CVID) phenotype. Front Immunol. 2019;10:1914.

71. Shulzhenko N, et al. CVID enteropathy is characterized by exceeding low mucosal IgA levels and interferon-driven inflammation possibly related to the presence of a pathobiont. Clin Immunol. 2018;197:139-153.

72. Kramski M, et al. Novel sensitive real-time PCR for quantification of bacterial 16S rRNA genes in plasma of HIV-infected patients as a marker for microbial translocation. J Clin Microbiol. 2011;49(10):3691-3693.

73. Eisenhofer R, et al. Contamination in low microbial biomass microbiome studies: issues and recommendations. Trends Microbiol. 2019;27(2):105-117.

74. Kozich JJ, et al. Development of a dual-index sequencing strategy and curation pipeline for analyzing amplicon sequence data on the MiSeq illumina sequencing platform. Appl Environ Microbiol. 2013;79(17):5112-5120.

75. Bolyen E, et al. Reproducible, interactive, scalable and extensible microbiome data science using QIIME 2. Nat Biotechnol. 2019;37(8):852-857

76. Callahan BJ, et al. DADA2: High-resolution sample inference from Illumina amplicon data. Nat Methods. 2016;13(7):581-583.

77. Pedregrosa F, et al. Scikit-learn: machine learning in python. J Mach Learn Res. 2011;12:2825-2830.

78. Wickham H, eds. ggplot2: Elegant Graphics for Data Analysis. Springer; 2016 